\title{
Intravenous Hydrogen Peroxide for Chronic Pain: An Alternative Etiology and Treatment Plan for the Long-Standing Painful States
}

\author{
Stuart L. Weg \\ Former Address: Department of Anesthesiology, Bergen Region Medical Center, Paramus, USA \\ Current Address: Patients Medical, New York, USA \\ Email: StuartLW@aol.com
}

Received 23 January 2014; revised 23 February 2014; accepted 27 March 2014

Copyright @ 2014 by author and Scientific Research Publishing Inc.

This work is licensed under the Creative Commons Attribution International License (CC BY).

http://creativecommons.org/licenses/by/4.0/

(c) (i) Open Access

\begin{abstract}
Background: Anecdotal reports of improvement in chronic pain patients given oxidative therapy (OXT) in the form of Intravenous Hydrogen Peroxide (IVHP) led the author to try this therapy on intractable pain cases. Method: 54 intractable non-malignant pain cases selected at random (16 had spinal problems, 14 had Interstitial Cystitis, 8 had headaches and 16 with miscellaneous problems) each received an average of 9 weekly IVHP infusions of $250 \mathrm{ml} 0.03 \%$ Hydrogen Peroxide. After 4 months of this therapy all patients were given a questionnaire to evaluate and study any improvements in their condition. The questions covered three areas: 1) Subjective improvements: pain, function, mental and emotional status, general health, and sexual health, 2) Analgesic medication usage: dosage and efficacy changes, 3) Satisfaction with the therapy and desire to continue. Results: $52 \%-70 \%$ mean $66 \%$ of the patients responded positively (more than slightly improved) to questions in the subjective areas, $85 \%$ of the patients were using less medication and/ or getting more effect from the analgesics they were taking, $96 \%$ of the patients wished to continue the therapy. These improvements were constant over the range of the four diagnostic groups. The improved patients all demonstrated some signs and lab results including fever, chills, abscess formation, purulent discharges, elevated WBCs and ESRs consistent with resolving systemic and/ or regional infections. Conclusions: IVPH can significantly improve patients in a majority of chronic painful conditions. Chronic Infection is associated with or absolutely the cause of Chronic Pain in the patients studied.
\end{abstract}

\section{Keywords}

Intravenous Hydrogen Peroxide (IVHP), Chronic Pain, Oxidative Therapy (OXT), Infectious Disease 


\section{Introduction}

In 1920 0.03\% Hydrogen Peroxide was given IV as a cure for Influenza infections occurring among British troops in India [1]. 0.03\% IV $\mathrm{H}_{2} \mathrm{O}_{2}$ was studied in the 1940's in the 1980's by Farr and others [2]-[5] Anecdotal reports on IVHP relate improvement in Migraine, Arthritis and other painful conditions. The author therefore administered $0.03 \%$ IVHP to a wide variety of patients who suffered intractable chronic pain of various etiologies.

\section{Method}

78 patients ages 20 - 74 with non-malignant, non-life threatening intractable pain who had failed all forms of conventional therapies were administered IVHP consisting of intravenous infusions of D5W containing $0.03 \%$ hydrogen peroxide following the Genesis Medical Center Protocol [2]. These infusions were given approximately once weekly. The Genesis treatment protocol called for a series of 10 - 20 infusions. All patients who received IVHP were requested to fill out the following questionnaire.

54 patients who received a total of 503 infusions in this study returned the questionnaire. 16 suffered pain from herniated cervical or lumbar spinal discs (HD) either with or without surgery. 14 patients had a diagnosis of Interstitial Cystitis (IC) some with removal of the bladder. 8 patients had intractable headaches (HA). Another group of 16 patients with various diagnosis associated with pain were labeled miscellaneous (Misc.) including TMJ, Arthritis, Multiple Sclerosis, Chronic Fatigue, Chronic Reflex Pain Syndrome etc. The average patient received 9.3 infusions with the range of number of infusions being 3 to 18 .

The survey questions were designed to evaluate changes in their pain, function, and medication use. Several questions also looked at mental health, sexual function, general health, and well-being. Two questions asked how the patients felt about this therapy and if they would do it again or continue.

The study was seeking to identify significant improvement. In order to register a positive response for significant improvement a patient had to score a number on a scale (see below) of 0 to 4 which indicated more than slightly improved. 0 and 1 were scored as negative, 2, 3 or 4 as positive.

\section{Questionnaire}

August 25, 1995

\section{Dear Patient,}

In the interest of sharing knowledge with the medical community, I have been asked to present a summary of the effects of Intravenous Hydrogen Peroxide on patients in pain. This is to take place at the annual meeting of the American Academy of Pain Management next month in Dallas Texas. The experiences that we have shared with this therapy are to be put in a poster form. I am therefore asking you to answer the following questions as accurately as possible and return the results to me at your first opportunity.

Please be advised that your name will appear only as your first name and an initial. The reason for this is to help me rather than for any identity purposes. No information about you personally other than clinical data will be used. Your identity will not be known.

When answering questions about how you are feeling in relation to the Peroxide treatments, please take a moment and reflect on the past as best you recall; ask a family member or close friend about those times.

Think about the number of bad days or good days.

Recall what your day was like, the activities that you were able to do.

How was your comfort level?

Did the medication for pain do the job then?

How were the nights? Your sleeping?

When relating the way you were, to how you are now please do not answer based on how you feel are at this specific moment. Give an answer that takes into consideration the overall change if any in your condition. Most pain patients taking Peroxide have had a rocky course. I know there have been ups and downs,

\section{But:}

Are you going in any direction?

Have you noted changes? Better or worse?

Are you doing more?

Have you been able to return to work, etc.?

Do you use more or less medication on an average?

Does the medication work better or worse?

Are there any changes in sleeping, walking, moving, voiding etc.? 
Are the flares as severe? As frequent?

I have developed the following 0 to 4 scale to help you classify your answers. Please put only one number as an answer. Here are the meanings of the numbers.

Number Description of your condition

Your condition/situation etc. is:

$\mathbf{0}=$ either worse or absolutely the same as before I got treated with Hydrogen Peroxide.

1 = no worse to slightly improved from before I got treated with Hydrogen Peroxide.

2 = slightly to moderately improved from before I got treated with Hydrogen Peroxide.

3 = moderately to greatly improved from before I got treated with Hydrogen Peroxide.

4 = greatly improved to in full remission or cured from before I got treated with Hydrogen Peroxide.

\section{Questions}

Using the above scale to respond to these question:

1. How is your worst or main pain? The one you came to me to treat?

2. How is your overall pain?

3. How is your ability to function (do things)?

4. How are other physical health complaints not related (as you know) to your pain? Examples: skin conditions, breathing, allergies, colds, urination,

5. How is your mental health? Examples, Emotional depression, thought process, focus and concentration, outlook, sleeping, etc.

6. How is your sexual function/drive?

7. How is your overall state of health or well-being?

Answer the next questions with yes or no only please.

8. Is the pain medication you use more effective?

9. Are you using less of it (or the same amount of a weaker type)?

10. Do you think that any benefit that you might have gained from Hydrogen Peroxide was worth the trouble and/or side effects that you suffered (if any)?

11. Would you do it again?

12. If not, would you do it again if the unpleasant side effects were better controlled or not present?

If you think there are reasons that your answers should not be included in this summary study please write them at the end of this page.

If you would like to include a special comment that will be placed in a document titled, "Patients Remarks" and open to the view of the medical community at the conference in Dallas, please feel free to do so either at the end of this page or on a separate sheet. Your name is optional.

Finally, thank you for your time in helping me prepare this summary. I will relate to you the response of the medical audience upon my return from the meeting.

Yours truly,

Stuart L. Weg MD

\section{Results}

The patient responses to the questions were scored as follows:

Scored as No Significant Improvement:

Response 0 = either worse or absolutely the same-------------------------------negative for improvement

Response 1 = no worse to slightly improved--------------------------------------negative for improvement

Scored as Significant Improvement:

Response 2 = slightly to moderately improved-----------------------------------positive for improvement

Response 3 = moderately to greatly improved--------------------------------------positive for improvement

Response 4 = greatly improved to in full remission or cured---------------------positive for improvement from before getting treated with $\mathrm{IV} \mathrm{H}_{2} \mathrm{O}_{2}$.

The Graph in Figure 1 comes from responses to question \#1. It shows the results of IVHP on the percentage of patients reporting significant improvement from their Main Pain or the pain diagnosis that brought the patient to the doctor for management;

a) All patients who responded are shown in the right column of Figure 1.

b) The other columns to the left have patient responses to IVHP by presenting pain diagnosis. 


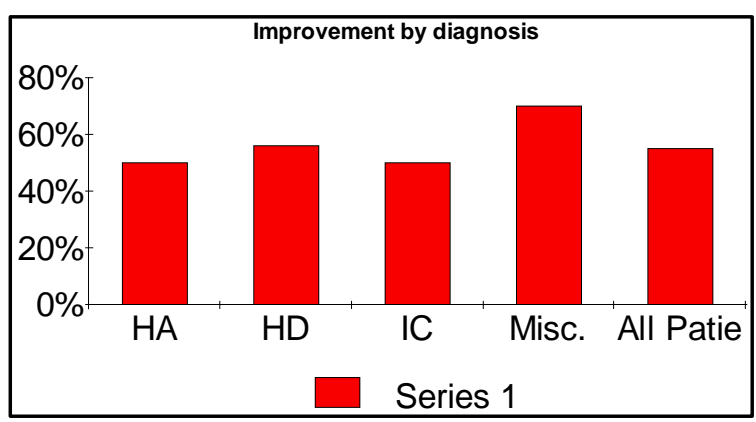

Figure 1. Percentage of individuals with Significant Improvement; Results of significant improvement broken down by Diagnosis. Headache (HA): $50 \%$; Herniated Discs (HD): 56\%; Interstitial Cystitis (IC): 50\%; Miscellaneous: 70\%; All Patients: 55\%.

The Graph in Figure 2 comes from patient responses to questions \#2 to \#8 (Categories of symptom complaints).

This graph shows the results of IVHP on the percentage of patients reporting significant improvement to the individual symptoms described in the questions. These include overall pain, function, other medical problems, mental, sexual and the feeling of well being.

The last two columns on the right are responses of significant improvement in a reduction of pain medication and a mean calculation of all responses.

The Graph in Figure 3 is from responses to questions \#8, 9 and 11.

This left graph shows the results of IVHP on the percentage of patients reporting significantly less pain and/or using less medication.

This right graph shows the results of IVHP on the percentage of patients and wanting to continue treatment.

\section{Summary}

As shown in Figure 1, about two thirds of the studied patients had significant improvement in their pain regardless of the diagnosis. From Figure 2 it is noted that this improvement rate was similar for all the associated problem areas that were questioned. From Figure 3 it is noted that nearly all patients had a reduced need for pain medication and wished to continue the treatment.

IVHP administration for pain is responsible for the following in this study:

1. Reduction of pain

2. Reduction of analgesics

3. Improved Function

4. Disappearance of depression

5. Improvement in mental performance

6. Improvement in health and well being

7. High satisfaction and desire to continue treatment

Most patients did get some mild flu like reaction. A few became ill with problems related to resolving infections. Some of these patients required admission to the Hospital or Emergency Department for observation. Reactions included septic-febrile reactions, urinary symptoms with bacteria, pulmonary congestion, abdominal cramping, diarrhea and vomiting. A number of patients had discharges of cultured pathogens in obviously septic bodily fluids. It was common to see scars erupt and exude such material. Painful fused shoulders and laminectomy scars in failed cases opened and drained in a similar fashion. Troublesome areas such as painful bulging discs in non-surgical cases developed hematin discoloration and eruption below the surface. In every case this corresponded to clinical improvement in the pain from that area. Because of these phenomena the patients became as convinced as we were that they were ridding themselves of an old deep infection.

\section{Conclusion}

This study demonstrated overwhelming evidence that IVHP is of therapeutic value in the treatment of a wide 


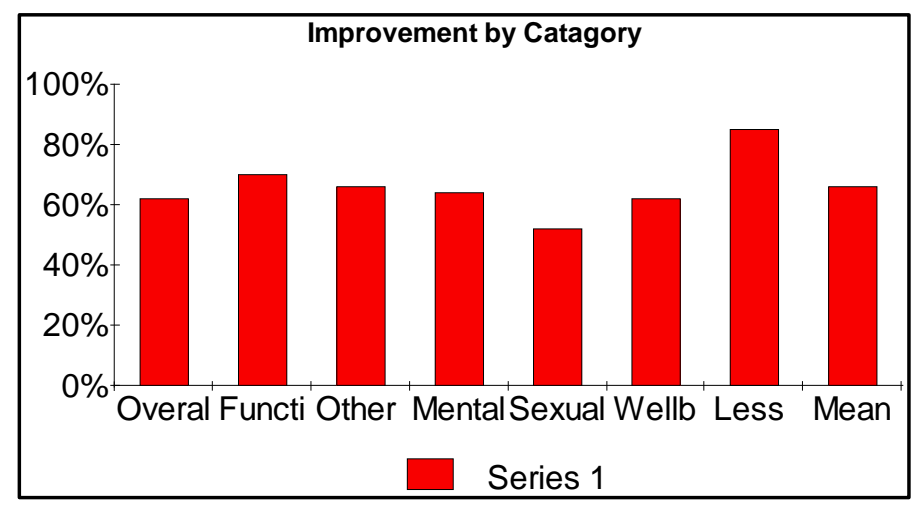

Figure 2. Significant improvement by each category of symptom complaints, reduction in medication and the Mean response. Symptom complaints with significant improvement: Overall Pain: 62\%; Function: 70\%; Other Medical Problems: 66\%; Mental Improvement: 64\%; Sexual Improvement ${ }^{* *}$ : 52\%; General Well-being: 62\%; Medication Benefit: 85\%; Mean Improvement: 66\%. ${ }^{* *}$ Male and female percentages were the same.

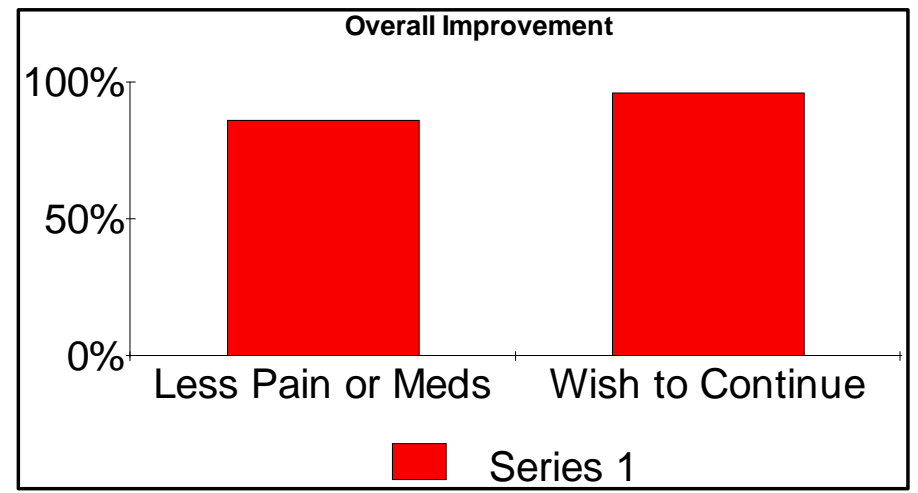

Figure 3. Percentage of patients with less pain and/or reduced medication use and percentage of patients wishing to continue this therapy. Significantly using less medication and/or feeling less pain: $88 \%$; Patients wishing to continuing treatment: $96 \%$.

range of intractable pain problems. The fact that IVHP is a known anti-infective treatment strongly suggests the existence of bacterial, fungal, mycoplasm etc. as a factor in intractable pain. This is further supported by the clinical observation of purulent bacterial shedding in nearly every patient either in the urine, head and neck secretions, scars, old wounds etc. This shedding always correlated to an eventual successful treatment.

There was a predictable tendency for problems to come to the surface with this treatment. Purulent drainage from previously closed wounds and old scars, septic episodes and bladder infections all developed and passed. Stitch and surgical sites developed small pustulates. When reactive areas that had not been thought of as troublesome erupted, it was then a diagnostic challenge to relate a past history of problems. One patients who had Interstitial Cystitis developed heel pain in a joint previously injected with steroids. Because of these reactions a number of patients temporarily suspended and in one case stopped treatment short of full benefit. There were no long term sequella in any patient.

1. Bacteria in urine, and purulent drainage

\section{Presenting Evidence of Infection}

2. Abscess formations over painful sites

3. Systemic symptoms, e.g.: fever, sweats, purulent discharges, fatigue

4. Local reaction over painful areas (e.g.: herniated discs) redness, pain, hematin

5. Elevated WBC’s, Diff shifts, increased ESR 


\section{Discussion}

As a result of this study, it is clear to the author that:

1. IVHP is effective for the treatment for a wide range of chronic painful conditions and their associated symptoms.

2. Chronic Infection is associated with or absolutely the cause of Chronic Pain in the patients studied.

This study was conducted in 1995 and presented as a poster in Dallas Texas in 1995. McMinn [6] noted and reported on that presentation in an article she wrote that year.

Ongoing clinical work and personal observations by the author since then have demonstrated that substituting other anti-infective oxidative or antibiotics therapies achieves similar results.

The recent reports of Albert [7] and others identify bacterial spinal infections associated with back pain; a double blind study also by Albert [8] supports the use of antibiotic therapy for back pain. The 1995 results described in this study which identify the efficacy of IVHP for Pain are important because IVHP is historically an anti-infective therapy and therefore supports the conclusions of Albert's work.

This study expands on the theory of Albert and should be considered with the current interest of infection causing chronic back pain.

Of further interest is that all patients with all forms of chronic pain not just back pain achieved similar responses to IVHP. This implies that the infections discovered by Albert may not be limited anatomically to Spinal pain and mobic changes. Based on the responses in this study of chronic pain patients to IVHP, the author has shown that infections are present and in all sites of chronic pain studied. That all of these diverse pain sites responded similarly to the broad spectrum anti-infective activity of IVHP, supports the author's belief that chronic pain is an infectious disease. Future studies on chronic pain should be aimed at the discovery of its origin in the context of an infectious disease.

\section{References}

[1] Oliver, T.H., Cantab, B.C. and Murphy, D.V. (1920) Influenzal Intravenous Injection of Hydrogen Peroxide. Lancet, 1, 432-433. http://dx.doi.org/10.1016/S0140-6736(01)11118-9

[2] Farr, C.H. (1987) The Therapeutic Use of Intravenous Hydrogen Peroxide (Monograph). Genesis Medical Center, Oklahoma City.

[3] Lorinez, A.L., Jacoby, J.J. and Livingstone, M.M. (1948) Studies on the Parenteral Administration of Hydrogen Peroxide. Anesthesiology, 9, 162-174. http://dx.doi.org/10.1097/00000542-194803000-00006

[4] Farr, C.H. (1989) Conference Presentation. First International Conference on Bio-Oxidativc Medicine. Dallas.

[5] Farr, C.H. (1988) Possible Therapeutic Value of Intravenous Hydrogen Peroxide. Plzen.lek Sborn., Suppl. 56, $171-173$.

[6] McMinn, M. (1995) I.V. Hydrogen Peroxide Touted for Intractable Pain, Suggesting an Infectious Component. Anesthesiology News, 4.

[7] Albert, H.B., Lambert, P., Rollason, J., Sorensen, J.S., Worthington, T. and Pedersen, M.B. (2013) Does Nuclear Tissue Infected with Bacteria Following Disc Herniations Lead to Modic Changes in the Adjacent Vertebrae? European Spine Journal, 22, 690-696. http://dx.doi.org/10.1007/s00586-013-2674-z

[8] Albert, H.B., Manniche, C., Sorensen, J.S. and Deleuran, B.W. (2013) Antibiotic Treatment in Patients with Low-Back Pain Associated with Modic Changes Type 1 (Bone Oedema): A Pilot Study. European Spine Journal, 22, 697-707. http://dx.doi.org/10.1007/s00586-013-2675-y 